\title{
En torno a la construcción social de la locura. Ian Hacking y la historia cultural de la psiquiatría*.
}

On the social construction of madness.

Ian Hacking and the cultural history of psychiatry.

\section{Rafael Huertas a.}

${ }^{a}$ Centro de Ciencias Humanas y Sociales CSIC.

Correspondencia: Rafael Huertas (rafael.huertas@cchs.csic.es)

Recibido: 14/03/2011; aceptado: 05/04/2011

RESUMEN: El objetivo de este trabajo es analizar la contribución del filósofo de la ciencia Ian Hacking a la historia cultural de la psiquiatría. Partiendo de conceptos propuestos por el autor, como "enfermedad mental transitoria" o "inventar/construir gente", se reflexiona en torno a la construcción socio-cultural de la enfermedad mental. Se examinan y discuten los dos estudios de caso propuestos por Hacking: la fuga disociativa y la personalidad múltiple, identificando las fortalezas y debilidades de sus planteamientos y la posible aplicación de los mismos a la historia y a la teoría psicopatológica.

PALABRAS CLAVE: Ian Hacking, Historia de la Psiquiatría, enfermedad mental transitoria, fuga disociativa, personalidad múltiple, constructivismo social.
ABSTRACT: The aim of this paper is to analyze the contribution of philosopher of science Ian Hacking to the cultural history of psychiatry. Based on ideas proposed by this author, as "transient mental illness" or "making up people", some reflections on the socio-cultural construction of mental illness are offered. The examination and discussion the two case studies proposed by Hacking, dissociative fugue and multiple personality, allow to identify the weaknesses and strengths of his approaches and its applicability to the history and theory of psychopathology.

KEY WORDS: Ian Hacking, History of Psychiatry, transient mental illness, dissociative fugue, multiple personality, social constructivism.

"A los alienistas del Pisuerga”

\section{Introducción}

Aunque con antecedentes notables $(1,2)$, en los años ochenta y noventa del siglo XX el estudio histórico de la enfermedad desde la perspectiva del constructivismo social adquirió una indudable preponderancia historiográfica $(3,4,5)$. Charles Rosenberg, uno de los más claros exponentes de esta tendencia en el ámbito de la historia de la medicina, llamó la atención sobre la complejidad de cualquier reflexión en torno a la enfermedad, que pretenda ir más allá de la mera consideración

\footnotetext{
* Trabajo realizado en el marco del Proyecto de Investigación HAR08-04899-C02-01, financiado por el Ministerio de Ciencia e Innovación.
} 
de una alteración anatómica o fisiopatológica del organismo humano. Para este autor, la enfermedad es "un acontecimiento biológico", pero también "un peculiar repertorio generador de construcciones verbales que reflejan la historia intelectual e institucional de la medicina, un aspecto de la política pública y la legitimación potencial de ésta, un elemento potencialmente definidor de roles sociales, una sanción de normas culturales y un elemento estructurador en las interacciones médicopaciente". Y concluye apuntando que "de alguna manera, la enfermedad no existe hasta que hemos acordado su existencia, al percibirla, nombrarla e intervenir sobre ella" (6).

Las enfermedades que etiquetamos como tales, no constituyen, según dicho acercamiento teórico, entidades "naturales" transhistóricas, que se mantienen en el espacio y el tiempo sino que son entendidas como "construcciones" intelectuales que se desarrollan en contextos sociales y culturales concretos. En este sentido, la enfermedad como objeto clínico únicamente existiría dentro del marco históricocultural en el que se constituye como entidad específica, por lo que sólo será plenamente comprensible desde su interpretación en dicho marco de referencia. Pero si esto puede ser aplicable a cualquier enfermedad, y pueden encontrarse en la historiografía ejemplos suficientemente concluyentes -desde la peste (7) al sida (8), pasando por la clorosis $(9,10)$ o la sífilis (11) -, en el caso de las enfermedades o los trastornos mentales, la reflexión en torno a "la locura construida" se enriquece de manera considerable, no solo por su enorme fuerza simbólica y estigmatizadora, que en buena medida comparte con las enfermedades infecciosas, sino por su propio carácter maleable y en cierto modo inasible de "lo otro de la razón". Además, las clásicas obras de Michel Foucault (12), o de Thomas Szasz (13), ya habían relativizado la propia existencia de la enfermedad mental antes de que el constructivismo social llegara a aplicarse con todas sus consecuencias a la historia de la medicina, mientras que George Rosen, a finales de los años sesenta, proponía abordar el trastorno psíquico "desde un amplio punto de vista, considerándolo fundamentalmente dentro del contexto de la comunidad, y teniendo en cuenta las estructuras y factores politicos, sociales y administrativos que han guardado alguna relación con la enfermedad mental en diferentes periodos históricos. Este estudio examina el reconocimiento de dicha enfermedad como problema de la comunidad, y las circunstancias que provocan dicho reconocimiento; los conceptos, ideas y teorías utilizables para interpretar una conducta extraña como enfermedad psíquica y que proporciona una base para la acción o inactividad de la comunidad; y el desarrollo y existencia de instituciones especiales para hacer frente a estos problemas" (14).

Si aceptamos esta posición, que en los años de la Guerra Fría hubiera sido calificada de "externalista" (15), tendremos que convenir que la enfermedad en general -y el trastorno mental en particular- es reconocida a través de su representación, 
ORIGINALES Y REVISIONES

es decir, por su descripción como "especie morbosa" o por la etiqueta diagnóstica que en nuestra cultura permita su identificación, y que dicha representación está sujeta a modificaciones diversas por las razones más variadas: científicas, sociales, políticas, culturales, etc. Como es bien sabido, este tipo de acercamientos y análisis han sido habituales en la historiografía psiquiátrica de las últimas décadas y han contribuido, en mayor o menor medida, a configurar un cierto "estilo de pensamiento" crítico en torno a las teorías y prácticas psi.

A finales de los años noventa, el filósofo de la ciencia Ian Hacking $(16,17)$, a través de dos estudios de caso concretos: la personalidad múltiple (18) y el automatismo ambulatorio o fuguismo (19), y de la propuesta de una categoría de análisis a la que denominó "enfermedad mental transitoria", introdujo nuevos elementos de discusión sobre la locura como construcción social o como elaboración cultural (20). Curiosamente, la propuesta de Hacking no venía del constructivismo, con el que se muestra distante aunque en modo alguno hostil $(21,22)$, sino de un realismo científico en sus intersecciones con la filosofía, la historia y la sociología (23). El propio Hacking se autodefine del siguiente modo: "me pienso como un "nominalista dinámico' interesado en cómo nuestras prácticas de nombrar interactúan con las cosas que nombramos, pero también podría decirse que soy un 'realista dialéctico' preocupado por las interacciones entre lo que existe (y lo que viene a ser) y nuestras concepciones de ello" (24). Más adelante retomaremos el concepto de interacción (o clase interactiva), baste ahora señalar que independientemente de cómo Hacking prefiera identificarse, su preocupación no es tanto profundizar en la naturaleza real o construida de las enfermedades mentales (transitorias), sino en las condiciones de posibilidad que deben cumplirse para que una enfermedad de este tipo llegue a diagnosticarse. Tal planteamiento puede tener, como no podía ser de otro modo, un gran atractivo para la historia y la epistemología de la psiquiatría porque nos permite reflexionar sobre la naturaleza de ciertos trastornos mentales en cualquier momento histórico -también en el presente-, así como sobre aspectos teóricos y prácticos de indudable trascendencia en la clínica y en el propio saber psicopatológico. Mi objetivo en las páginas que siguen es analizar la contribución de Hacking en relación a las "enfermedades mentales transitorias", valorar sus fortalezas y debilidades, y reflexionar sobre la potencialidad de su propuesta metodológica y su posible aplicación a las investigaciones histórico-psiquiátricas.

\section{Las enfermedades mentales "transitorias"}

Hacking define la "enfermedad mental transitoria" [transient mental illnes] no en términos individuales, no como un trastorno o dolencia que tenga una du- 
ración limitada, apareciendo y remitiendo durante la vida de un sujeto, sino en un sentido colectivo e histórico: aquella que aparece en un tiempo y un lugar determinado y, o bien desaparece sin dejar rastro o bien reaparece en otro lugar y en otras circunstancias, siempre por razones que tienen que ver con el ambiente cultural de la época y del país o contexto socio-geográfico en el que la enfermedad surge como tal. Para localizar e identificar dichas enfermedades transitorias recurre a la metáfora del "nicho ecológico", entendiéndolo como un lugar suficientemente amplio en el que se den las condiciones ambientales adecuadas para que la enfermedad (o el síntoma) pueda desarrollarse. En el interior del mencionado nicho actuarían una serie de vectores que desenvolviéndose en varias direcciones, terminarían por generar como resultante la aparición de la enfermedad mental.

Los cuatro vectores que, según Hacking, ejercerían en el interior del nicho ecológico de la enfermedad mental son los siguientes:

1) vector lingüístico-taxonómico: la "nueva enfermedad" debe encajar en algún sistema taxonómico, debe ocupar un lugar específico en el contexto de una determinada clasificación nosográfica ya establecida, debe poder ser nombrada y reconocida de acuerdo con el lenguaje científico al uso.

2) vector de polaridad cultural: la enfermedad debe ubicarse entre dos elementos, entre dos polos culturales antitéticos: lo malo y lo bueno; el vicio y la virtud, siendo a la vez objeto de admiración colectiva y de testimonio de un comportamiento degradado que crea inquietud o repulsión. Obviamente, las valoraciones éticas de la bondad o la maldad están sujetas a variaciones históricas, lo que supone una complejidad y un interés añadido.

3) vector de observabilidad: la enfermedad ha ser "visible" e identificable como desorden y/o como sufrimiento, como "comportamiento patológico" en suma, tanto para los expertos como para la población en general.

4) vector de liberación-agregación: dicho comportamiento patológico debe permitir alcanzar objetivos vitales que sería imposible alcanzar de una manera normalizada, lo que ayudaría, como más tarde intentaré explicar, al reclutamiento social de los individuos que padecen la enfermedad.

Hacking intenta demostrar su modelo "vectorial" en los locos viajeros (Mad Travelers), esto es, aquellos sujetos que desaparecen de su domicilio y de su puesto de trabajo para deambular -vagabundear- y desplazarse, recorriendo a veces grandes distancias, llegando a otros países y regresando al cabo de un tiempo para, en ocasiones, volver a marcharse sin previo aviso (19). Dicha actitud, fue descrita por primera vez por Philippe Tissié en 1887 (25), dio lugar a una amplia producción científica en torno a una nueva patología, reconocida como tal por la comunidad científica y por la opinión pública, que en un primer momento recibió el nombre de automatismo ambulatorio (26). El debate sobre la "naturaleza" de la nueva enfermedad fue intenso en las últimas décadas del siglo XIX. Mientras algunos autores 
ORIGINALES Y REVISIONES

apuntaron su origen histérico $(27,28)$, Charcot y sus colaboradores de la Salpêtrière la clasificaron como una subclase de la epilepsia $(29,30)$. Independientemente de la discusión suscitada, lo que interesa señalar aquí es que el llamado automatismo ambulatorio pudo incluirse en una taxonomía ya establecida. Histérico o comicial, el nuevo cuadro podía considerarse tan real, tan natural, como la histeria o la epilepsia, porque no era sino una variedad de una enfermedad suficientemente admitida y reconocida (19). Incluso cuando se describió la dromomanía como un tipo de fuguismo que no cabía en ninguna de las categorías antedichas, tampoco suscitó un grave problema taxonómico, pues fue catalogada en el marco de la degeneración (31).

Se trataba, además, de un comportamiento patológico capaz de suscitar una incómoda fascinación que pivotaba entre la desconfianza y el temor hacia los vagabundos, por peligrosos o molestos, y la atracción hacia los viajes y los viajeros. En este sentido, hay que destacar que la formulación de la nueva categoría diagnóstica y el debate científico subsiguiente tiene lugar entre 1887 y 1900, coincidiendo con un fenómeno hasta entonces inédito: el gusto por los viajes por parte de la clase media. El esplendor de la Gare Saint-Lazare, inmortalizada por Monet en 1877, o las novelas de viajes y aventuras de Stevenson, Mark Twain o Jules Verne son fiel reflejo de unas condiciones sociales y culturales que no debieron ser ajenas al surgimiento del viajero patológico. Pero, ¿por qué en Francia y no en otros contextos geográficos con unas condiciones locales similares como Inglaterra o Estados Unidos? Porque en esos otros lugares -nos dirá Hacking- no se pueden identificar ni el vector de observabilidad, ni el de polaridad cultural. En el primer caso, y al contrario que en Francia $(32,33,34)$, ni en Inglaterra ni en Estados Unidos se reclutaba de manera obligatoria a los soldados, por lo que no había un cuerpo de médicos forenses que investigara sistemáticamente a los viajeros en busca de desertores. En el segundo, el vagabundaje no constituía un problema social en ninguno de los dos países sajones, acostumbrados a que sus hombres se ausentaran marchando a ultramar o a territorios por explorar y colonizar.

La elección del automatismo ambulatorio por parte de Hacking para ilustrar su modelo de "enfermedad mental transitoria" es, sin duda, muy acertada, pues su "nicho ecológico" queda perfectamente articulado con sus cuatro vectores bien definidos. Es posible que dicho modelo no llegue a cumplirse con tanta precisión en otros trastornos mentales pero no cabe duda que su propuesta ofrece elementos importantes de reflexión en torno a la construcción o elaboración cultural de los mismos. Por citar un ejemplo muy extremo, en 1850, la Medical Association of Lousiana reconoció un cuadro "psiquiátrico", que se denominó drapetomanía [drapetes: esclavo fugitivo], en algunos esclavos cuyo "síntoma" fundamental era el deseo "irracional" de huir de las plantaciones $(35,36)$. Como es lógico hoy se considera una muestra de racismo científico, pero es obvio que dicha "enfermedad" 
fue nombrada con un término científico reconocible y ubicada en el ámbito taxonómico de la monomanía, observada y aceptada en un lugar concreto -los estados del sur-, situado entre dos polos antitéticos: esclavismo versus abolicionismo; y con un objetivo de libertad, incompatible con la condición "natural" del esclavo.

Pero no hace falta recurrir a casos tan excesivos o tan limitados. Recientemente, Emilio Vaschetto ha estudiado las locuras puerperales en Argentina desde la perspectiva que nos ocupa, al comprobar la frecuencia con que el diagnóstico de psicosis puerperal aparece en los archivos clínicos de maternidades y manicomios argentinos en el tránsito del siglo XIX al XX, así como la gran cantidad de obras (tesis doctorales y publicaciones científicas) dedicadas a dicha patología. Desde el punto de vista taxonómico, la psicosis puerperal fue considerada una "locura tóxica" e incluida en las nosografías junto al morfinismo y otras "intoxicaciones", con la particularidad de que se trataba de una toxicidad endógena, generada en el interior del cuerpo femenino. Los dos polos culturales vendrían definidos por un cierto sentimiento de conmiseración hacia la mujer embarazada o puérpera que enloquecía y por el rechazo social hacia unas mujeres que o eran extranjeras o pertenecían a grupos marginales (prostitutas o vagabundas). Finalmente, la observabilidad vendría en este caso, condicionada por los cambios en la intimidad del momento del parto: del ámbito doméstico al hospitalario (37). Es evidente que el parto en los hospitales públicos de la beneficencia facilitaba la visibilidad de la patología puerperal en las mujeres pobres y también su absorción en el discurso no solo psiquiátrico, sino también obstétrico (38).

El trabajo de Vaschetto es interesante porque la locura puerperal no era una "nueva enfermedad". Había sido descrita a mediados del siglo XIX, siendo El Traité de la folie des femmes enceintes, des nouvelles accouchées et des nourrices de Louis Victor Marcé, publicado en 1858, una de las obras de referencia ineludibles. Sin embargo, en el último tercio del mismo, esta locura puerperal fue sometida a una amplia revisión que trajo consigo el cuestionamiento de la misma como una entidad nosológica distinta de las demás vesanias $(39,40)$. Por eso no deja de llamar a atención el "éxito" que este diagnóstico alcanzó en Argentina años más tarde. Serían necesarios estudios comparados que nos permitieran conocer si la psicosis puerperal apareció de manera tan abrupta y en la misma época, en otros contextos geográficos, sociales y culturales. También habría que valorar el proceso de recepción y de transmisión del conocimiento en el país y en el ámbito científico que nos ocupa; pero no cabe duda que el modelo hackiniano, aplicado de una manera no dogmática, le sirve al autor argentino para orientarse hacia el análisis de determinados parámetros que corroboran la psicosis puerperal como una construcción socio-cultural.

El modelo de las "enfermedades mentales transitorias" puede, pues, ser una herramienta útil para el estudio de los trastornos psíquicos, pero tampoco convie- 
ORIGINALES Y REVISIONES

ne elevarlo a la categoría de fetiche metodológico. Es verdad que la historia de la psiquiatría -y de la medicina- está repleta de diagnósticos desaparecidos, pero las razones pueden ser muy variadas: científicas (cambios de paradigma); prácticas (posibilidades técnicas), demográficas, ... y naturalmente, sociales y culturales, pero, aun siendo necesario - yo diría que obligatorio- una adecuada contextualización histórico social de la enfermedad o del síntoma que estemos estudiando, lo que puede explicarnos su aparición en las nosografías, tampoco podemos caer en la ingenuidad de aceptar su "desaparición" absoluta sin valorar antes su posible evolución conceptual, su cambio de nombre o su adaptación a nuevas sistemáticas. Pongamos dos ejemplos suficientemente significativos de lo que Hacking entiende por enfermedades transitorias: la monomanía y la histeria. Ambos diagnósticos estuvieron sujetos, no cabe duda, a una elaboración cultural sin precedentes. Incluso, si nos esforzamos, podríamos identificar algunos de los vectores descritos por el autor canadiense. Así, en cuanto a la monomanía, es sabido que en el plano lingüístico-taxonómico los problemas médico-legales suscitados por la "manía sin delirio" de Pinel, facilitaron la aparición de una nueva entidad capaz de explicar adecuadamente el "delirio parcial". El problema terminológico que se planteaba era la necesidad de entender el concepto "manía sin delirio" de forma contraria a su significado semántico. Por eso la opción "manía sin delirio con delirio específico" fue sustituido por el de monomanía, para caracterizar el rasgo principal de la enfermedad; esto es, una idea fija que se hace dueña de la mente del sujeto $(41,42)$. Asimismo, "la doble ola de horror y atracción que rodeó a la figura sanguinaria y temida del delincuente, el enemigo primero de las pregonadas virtudes burguesas de la época" (43), puede ser aplicable al cleptómano, al pirómano o al monomaníaco homicida, y es una buena muestra de polaridad cultural. Digamos, finalmente, que a pesar de los enconados debates entre médicos y juristas $-\mathrm{y}$ en el seno del propio movimiento alienista- sobre la existencia real de la monomanía, lo cierto es que tal diagnóstico fue encontrando un grado de aceptación cada vez mayor en las salas de justicia (44); lo que debe interpretarse, sin duda, como una expresión del progresivo reconocimiento social en Francia de los alienistas como los grandes expertos en la locura (45). La monomanía se hizo "visible", aunque para su identificación fuera necesario el concurso del experto alienista capaz de reconocer y diagnosticar a los "locos que no lo parecen" $(46,47)$.

Algo diferente, aunque igualmente significativo, sería el proceso de construcción cultural de la histeria a finales del siglo XIX. Es imposible revisar aquí la amplia bibliografía existente sobre esta cuestión (48), digamos simplemente que la histeria no solo se hizo muy "visible", sino que impregnó la sociedad europea finisecular de tal modo que, aun reconociendo la existencia de "expertos" en la susodicha "enfermedad", todo el mundo, desde muy diversos sectores sociales, parecía tener algo que decir, hasta el punto de que los discursos médicos sobre la 
histeria estuvieron sometidos a las mentalidades, los códigos y los valores fin de siècle (49).

La histeria ya estaba "nombrada" desde la Antigüedad; pero existen elementos profesionales y políticos que permiten explicar la eclosión diagnóstica y cultural de la histeria en Francia a finales del siglo XIX (45), de algún modo comparable a lo ocurrido en Estados Unidos con la descripción de la neurastenia por G.M. Beard (50). Tampoco resulta difícil identificar la ambivalencia que puede existir ante la seducción manipuladora de la histérica, y la capacidad o el intento de conseguir sus objetivos vitales - de satisfacción del deseo- a través del síntoma.

Ahora bien, ¿pueden considerarse la monomanía y la histeria "enfermedades mentales transitorias", en el sentido de Hacking? Es cierto que ambas tuvieron formulaciones con un indudable componente de elaboración cultural y que, incluso, en esos momentos concretos, podrían identificarse sus nichos ecológicos y los vectores que operan en su interior; también podría argumentarse que tanto una como otra han dejado de "diagnosticarse", han dejado de ser reconocidas como tales en las nosografías al uso. No existen. Pero ¿han desaparecido sin dejar rastro?

La respuesta negativa a este interrogante me parece obvia. La monomanía esquiroliana se mantuvo como diagnóstico durante todo el siglo XIX, trascendiendo, además, las fronteras geográficas y culturales francesas (51). Su presencia puede rastrearse no solo en las obras de los alienistas, sino en célebres casos judiciales, como el de Pierre Rivière (1835), estudiado por Foucault (52), o como el ya mencionado de "El Sacamantecas" (1880) en España $(53,54)$. Convivió con el degeneracionismo (55), hasta que acabó siendo desplazado por este nuevo paradigma, más acorde con el determinismo de la ciencia positivista y más útil para las estrategias profesionales de los alienistas, ya que el somaticismo y los signos físicos aportaban argumentos "objetivos" a los peritajes psiquiátricos (56).

La monomanía acabó desapareciendo como etiqueta diagnóstica, pero el problema de las locuras parciales -o de las locuras razonantes- siguió siendo uno de grandes los caballos de batalla del alienismo. Cuando en 1909, Sérieux y Capgras describen el delirio de interpretación (57), reconocen su deuda con Esquirol y, en particular, con sus monomanías intelectuales. Monomanía, delirio de interpretación y paranoia se sitúan, pues, en una misma línea de desarrollo atravesada por cambios en el entorno socio-cultural. La propia paranoia tuvo dificultades de ubicación nosográfica sin que por ello pueda aventurase su "desaparición” (58).

El caso de la histeria es todavía más esclarecedor, es cierto que sus primeras formulaciones no dejaron de ser una fantástica construcción cultural propia de aquel fin de siècle, pero no lo es menos el que tras las correcciones oportunas, dio lugar a desarrollos de gran interés psicopatológico convirtiéndose en referencia ineludible para una nueva psicopatología de las neurosis. Hoy día ya no se ven los floridos ataques histerico-epilépticos descritos por Charcot, pero no puede olvidar- 
ORIGINALES Y REVISIONES

se que la histeria posibilitó la invención del psicoanálisis y que desde los enfoques más dinámicos se reivindica su vigencia y su utilidad tanto en la clínica como en la teoría psicopatológica $(59,60)$. Aún así, la histeria ha desaparecido paulatinamente de la nomenclatura psiquiátrica al uso, tanto la CIE-10 como el DSM-IV no la recogen en sus glosarios, por más que en este último pueda a duras penas subsistir en los llamados trastornos disociativos y en los trastornos somatomorfos. Obviamente, ningún nominalismo es inocente $y$, en este caso, refleja un fuerte componente ideológico y doctrinal.

Incluso el fuguismo, presentado por Hacking como gran estudio de caso en el que demostrar la existencia de enfermedades mentales "transitorias", ha pervivido en las nosografías psiquiátricas con modificaciones conceptuales más o menos ostensibles hasta ser incluido en el DSM-IV como fuga disociativa $(61,62)$.

Así pues, una cosa es que una enfermedad "desaparezca", y otra bien distinta, como ya he adelantado, que se produzca una evolución conceptual en su manera de entenderla o un cambio en la manera de nombrarla y de clasificarla. En este sentido, cabría preguntarse si el nicho ecológico de una enfermedad mental "transitoria" podría sobrevivir a la desaparición o modificación de algunos de los vectores que lo componen. La pregunta no es baladí, porque si se aceptara dicha circunstancia, la enfermedad mental dejaría de ser "transitoria"; lo cual no necesariamente invalidaría el planteamiento de Hacking sino que, paradójicamente, nos ofrecería más posibilidades de análisis, al poder considerar los famosos vectores de una manera menos estricta, menos categórica. Una opción podría ser entenderlos como "umbrales de entrada" -tal vez suficientes, aunque no necesarios- para la elaboración cultural de un trastorno mental. La anorexia nerviosa, por ejemplo, ha sido estudiada desde esta perspectiva (63), aun cuando el ayuno excesivo en las mujeres está documentado desde hace siglos $(64,65)$ y su descripción como patología mental fue realizada de manera casi simultánea por varios autores en la década de 1870 $(66,67)$, asimilándose a un forma de histeria con elementos "perversos". Un marco nosográfico y cultural bien distinto al del momento actual, en el que la vivencia del propio cuerpo adquiere unas connotaciones muy particulares (68) e, incluso, las nuevas tecnologías permiten novedosos estudios culturales impensables en otras épocas (69).

Así pues, me parece que la reflexión sobre la elaboración cultural de la locura a través de la propuesta de las "enfermedades mentales transitorias" va más allá de determinadas enfermedades que "aparecen y desaparecen". Lo que sí queda patente, y probablemente ésta es una de las aportaciones más aprovechables de Hacking, es la existencia de trastornos mentales que pueden considerarse como particularmente emblemáticos en un momento histórico determinado y en unas coordenadas culturales específicas. La construcción social o cultural de la enfermedad mental se nos presenta como un elemento fundamental para entender la clínica, para in- 
terpretar correctamente los cambios conceptuales y prácticos en torno al quehacer psiquiátrico y para comprender la actitud social hacia la locura y el loco.

Ahora bien, la descripción de nuevas enfermedades mentales (transitorias o no) requieren tanto víctimas como expertos; alienados y alienistas; enfermos y médicos o psicólogos.

\section{"Inventar/construir personas"}

Si en Mad Travelers se ilustraban las particularidades fundamentales de lo que Hacking denomina "enfermedades mentales transitorias", en Rewriting the Soul, se aborda el caso de la personalidad múltiple ofreciendo otras claves interpretativas que merece la pena analizar. El síndrome de personalidad múltiple es considerado también una enfermedad de carácter transitorio, surgida primero en la Francia de finales del siglo XIX, y ligado una vez más a la histeria (70). Tras años de olvido, el síndrome reapareció en Estados Unidos en los años 80 del siglo XX, produciéndose una verdadera "epidemia de múltiples" e incluyéndose oficialmente en el DSM-III. Pero, en esta ocasión su objetivo se amplía hacia otras facetas de la actividad diagnóstica: "El relato de la personalidad múltiple es, en buena medida, una narración acerca de lo que he llamado inventando personas. Estoy fascinado por la dinámica de la relación entre personas que se conocen, el conocimiento acerca de ellas, y los que las conocen" (18).

Hacking recurre de nuevo a la metáfora de los vectores para señalar dos características sobresalientes en el proceso que denomina "inventar/construir gente" [making up people] (71). El vector de etiquetado, por parte de los expertos, que crea una realidad que alguna gente hace suya, y el vector de la experiencia autónoma de la persona etiquetada, que a su vez recrea unas circunstancias que el especialista debe afrontar.

Es evidente que la pretensión normalizadora de la medicina (y psiquiatría), debidamente asistida por la estadística, facilitó la creación de nuevos espacios en los que enumerar y clasificar a las personas. No es que una determinada clase de sujetos ya existente comenzara a ser reconocida por los administradores o por los científicos expertos en la naturaleza humana, sino que esa clase de individuos "viene a ser" -se inventa, se construye- al mismo tiempo que la propia clase o categoría es formulada. Las clases, especies o variedades "naturales" (desde los electrones a los distintos rangos taxonómicos del reino animal, vegetal o mineral) son indiferentes, no cambian según la manera en que sean nombrados y no son afectadas por las prácticas culturales, pero las clases humanas son interactivas, su manera de ser y de actuar, su subjetividad y sus acciones no son independientes de cómo son des- 
ORIGINALES Y REVISIONES

critos y clasificados. Esto es lo que Hacking llama el efecto bucle [looping efect] de las clases humanas $(72,73)$ : las interrelaciones entre la gente y las formas en que ésta es clasificada. Las personas tienden a conformarse, a permanecer e incluso a crecer, en el ámbito clasificatorio en el que han sido descritos o diagnosticados.

La influencia de la escuela sociológica de Chicago, y su formulación de la teoría del etiquetado [labeling theory], es más de evidente. Hacking sigue a Erving Goffman (74), cuando advierte que ciertas categorías de individuos son creadas, no existen más que en el momento de ser definidas y estudiadas por sociólogos, criminólogos o psicólogos. Sin embargo, esas categorías, esas etiquetas, tienen efectos sobre las personas clasificadas; unas veces de manera directa, emanado de su propio conocimiento de haber sido catalogadas. En el caso de la personalidad múltiple, los elementos culturales y mediáticos favorecieron de manera importante que muchos pacientes asumieran para sí mismos esta condición: programas de televisión, revistas populares, películas, etc., en torno a la personalidad múltiple impregnaron la sociedad y la cultura norteamericanas de los años setenta y ochenta, poniendo de moda un tópico en el que la realidad de este trastorno mental se constituyó en el bucle creado por los que encarnan el conocimiento (los expertos), los conocidos (o etiquetados) y el propio conocimiento en sí.

Otras veces, el efecto bucle es indirecto, sobre todo cuando las clasificaciones son incorporadas al funcionamiento de las instituciones: la mayor parte de los criminales o de los locos no tienen ningún conocimiento de las clasificaciones criminológicas o psiquiátricas, no habría en estos casos un efecto bucle directo, pero sí efectos indirectos debido a la interacción con las instituciones y con los roles creados en la prisión o en el manicomio.

Esta interacción indirecta sería aplicable también, al autismo infantil. Los niños autistas -y otro tanto podría decirse de la "infancia anormal" en general (75) - no pueden conocer su clasificación ni interactuar con ella, pero en nuestro mundo de burocracia pedagógica y psicológica, los niños autistas son integrados en prácticas institucionales, la interacción y el efecto bucle se produce a nivel institucional (76).

También puede haber una redescripción o reformulación de ciertas situaciones antiguas a la luz de "conocimientos" modernos. Cabe pensar que si un cuadro clínico no existía o no estaba disponible en un tiempo pasado, resultaba imposible actuar sobre él. La situación pudo tener lugar pero no fue reconocida en su momento, al menos como patológica. La memoria desempeñaría aquí un papel fundamental, de modo que el pasado puede ser revisado retrospectivamente, cambiando la sensibilidad y la comprensión de los hechos. El pasado vuelve entonces repleto de acciones "intencionales" que no lo fueron en su momento. Esto es lo que ha ocurrido, según Hacking, con el abuso infantil, que se ha expandido de tal forma que más y más situaciones caen bajo su descripción y cada vez más hombres y mujeres 
acaban viéndose, retrospectivamente, a sí mismos como abusados/ maltratados o como abusadores/maltratadores (18).

Como se sabe, el abuso sexual infantil tiene unas conexiones muy directas con el desorden de personalidad múltiple o, si se prefiere, con el Trastorno de de Identidad Disociativo. Incluso ha llegado a entenderse dicho desorden como un mecanismo de defensa para poder sobrellevar el dolor o el miedo provocado por un abuso sexual repetido durante la infancia (77). No podemos dejar de recordar, a este respecto, la primera hipótesis de Freud sobre la etiología de la histeria y su revisión en torno a la teoría de la seducción y las relaciones entre trauma psíquico y memoria "construida".

En cualquier caso, lo que me interesa destacar aquí es que con este planteamiento se introduce un elemento de causalidad que viene a trastocar las tradicionales taxonomías basadas en agrupaciones de síntomas. Es evidente que si se sabe la etiología de una enfermedad, habremos dado un gran paso en su conocimiento y, además, el tratamiento (etiológico) tendrá muchas más garantías de éxito. Realmente, esto solo funciona en las enfermedades infecciosas o en los envenenamientos, en los que el antibiótico o el antídoto pueden ofrecer una eficacia inmediata; pero no tanto con los controvertidos "factores de riesgo" u otros elementos de causalidad que solo permiten dirigir estrategias profilácticas más o menos orientadas.

En los años ochenta del siglo XX, la literatura científica estableció la relación entre el abuso sexual infantil y la personalidad múltiple $(78,79,80)$, pero como es bien sabido correlación no implica causalidad. Es muy posible que el abuso sexual, concluye Hacking, sea un elemento importante, incluso imprescindible, para configurar el "estereotipo" de la enfermedad, pero la supuesta causalidad de la personalidad múltiple se acaba constituyendo como una etiología autoconfirmada en la que no cabría establecer la realidad de la enfermedad mental mediante la asignación de las causas (18).

Lo que no especifica Hacking, aunque de algún modo está implícito en su discurso, es que la propia noción de "abuso infantil" surge en esa época como una construcción social y cultural, no porque no existiera con anterioridad sino porque, de la mano de los derechos del niño, la sociedad en su conjunto percibe el maltrato infantil como un problema que necesita técnicos y expertos. En 1977 se fundó la revista Child Abuse \& Neglect-órgano de expresión de la Internacional Society for Prevencion of Chile Abuse and Neglect-, y sus páginas constituyen un interesante reflejo de la evolución de los discursos psico-sociales en torno al abuso infantil durante las últimas décadas. Esta sensibilidad social hacia la agresión sexual a los/las niños/as ha tenido, no cabe duda, efectos muy positivos en cuanto a la protección de la infancia, por más que se apuntara la posibilidad de que en ocasiones pudiera ignorarse, o pasar desapercibida, incluso en ámbitos especializados, produciéndose lo que se dio en llamar, creando un nuevo cuadro 
ORIGINALES Y REVISIONES

psicológico (esta vez de negación ante supuestas evidencias), el "síndrome de acomodación al abuso infantil" (81).

Tampoco podemos olvidar en este contexto, otras elaboraciones culturales, con un fundamento científico más que discutible, que llegaron a hacer fortuna o, por lo menos, han sido objeto de importantes debates, como el "síndrome de alienación parental”, descrito por Richard Gardner en 1985 (82), que coincidió con la promulgación de la Ley de custodia compartida en estados Unidos y su aplicación en las tramitaciones de divorcios. Como se sabe, dicho "síndrome" está siendo utilizado recientemente en España a raíz, igualmente, de la Ley de custodia compartida de 2005, llegándose a presentar como un nuevo trastorno psíquico. Antonio Escudero et al. han puesto de manifiesto las profundas debilidades metodológicas, así como las falacias que la formulación del síndrome de Gardner encierra (83), pero resulta obvio la importancia de elementos (vectores) culturales, y sus connotaciones legislativas (matrimonio y divorcio, custodia de los hijos, la visibilidad del maltrato de género y del abuso infantil, etc.), en la construcción de un supuesto cuadro clínico y en la invención de un persona portadora del mismo (84).

Como se ve, al igual que ocurre con las enfermedades mentales transitorias, el proceso de inventar/construir personas puede ir mucho más lejos de las coordenadas en las que Hacking sitúa sus ejemplos. En definitiva, podemos concluir que su propuesta en torno a la enfermedad mental aporta elementos de reflexión de gran interés para la historia de la psiquiatría porque viene a actualizar -y a problematizar- el debate sobre la construcción social/elaboración cultural de la locura. Como hemos visto, algunas de sus afirmaciones pueden resultar equívocas o discutibles desde el punto de vista historiográfico, pero debemos tener en cuenta que Hacking en ningún momento pretende hacer historia de la psiquiatría, sino profundizar, desde la perspectiva de la filosofía de la ciencia, en aspectos como la causalidad, el nominalismo, las clasificaciones o la elaboración de conceptos, la manera de nombrarlos y sus consecuencias sobre las personas. En este sentido, resulta evidente su conexión con el M. Foucault de Las palabras y las cosas o, como ya he apuntado, con E. Goffman, dos autores fundamentales y ya clásicos en la historiografía crítica de la psiquiatría (85).

La historia de la psiquiatría está repleta, en efecto, de diagnósticos que o han desaparecido o se han trasformado o ya no copan los dictámenes clínicos como lo hicieron en su momento. Sin embargo, ante el "descubrimiento" o, si se prefiere, la "invención", de una nueva entidad psiquiátrica, se nos abren al menos dos interrogantes. En primer lugar, ¿se trata de una enfermedad "transitoria", en el sentido de Hacking?, o por el contrario, ¿encontraríamos, a poco que nos esforcemos, antecedentes descriptivos en los clásicos de la psicopatología que nos orientasen sobre la "continuidad" o "metamorfosis" de determinados cuadros clínicos?. En segundo lugar, siempre cabe preguntarse si dichas manifestaciones clínicas res- 
ponden a procesos biológicos o biográficos concretos o si nos encontramos ante unos "síntomas" dependientes de movimientos sociales específicos; dicho de otra manera, ¿cuanto hay de elaboración cultural en un determinado trastorno mental? Esto no quiere decir, ni mucho menos, que se discuta la existencia del trastorno (o enfermedad) mental o de determinadas formas de alienación pero sí nos permite relativizar, desde una perspectiva filosófica y cultural, la sanción o el estatuto exclusivamente bio-médico de la misma. En definitiva, no me parece que el dilema deba ser establecerse entre enfermedad "real" y enfermedad "construida"; un edificio se construye, pero no por eso es menos real que una especie animal o vegetal -un producto de la naturaleza que, finalmente, acaba nombrándose, clasificándose y "construyéndose"-

Además de los trastornos ya analizados -la histeria, la monomanía, etc.-, otras "perlas" de la psicopatología más clásica pueden ser susceptibles también de este tipo de acercamientos. Pondré un solo ejemplo: la pasión erótica por las telas en la mujer descrita por Clerambault en 1908-1910 $(86,87)$, supone una muestra de la finura descriptiva del responsable de la Enfermería Especial de la Prefectura de Policía de París, pero responde también a unos condicionantes socio-culturales que vienen marcados, al menos en parte, por la modernidad urbana de las reformas de Haussmann en el Paris de la segunda mitad del siglo XIX. A partir de 1855 empiezan a funcionar los grandes almacenes (Les Magasins du Louvre en 1855; Le Bazar de l'Hôtel de Ville en 1856; Le Printemps en 1865; La Samaritaine en 1869 y Les Galeries Lafayette en 1895), muy pronto Lasègue describe la cleptomanía en estos nuevos espacios de consumo (88) y Clérambault "encuentra" la asociación entre cleptomanía y un cierto tipo de fetichismo que tiene que ver con la pasión erótica de algunas mujeres por las telas de seda robadas en esos grandes almacenes (89).

Incluso la propia esquizofrenia puede ser entendida como una perturbación de origen histórico-cultural. Pese a que el pensamiento psiquiátrico más hegemónico considera la esquizofrenia como una clase natural (como una enfermedad física), que curiosamente, el propio Hacking llega a aceptar (21), siguen existiendo argumentos en favor de su relación con el surgimiento de la conciencia moderna, de una cultura de la subjetividad, cuyo individualismo exacerbado y las nuevas formas de intimidad (y de interioridad), pueden inducir, cuando no imponer, la fragmentación del yo y la constitución de un trastorno característicamente moderno $(90,91,92)$.

Es evidente, y las páginas precedentes han intentado argumentarlo con insistencia, que la expresión social de la enfermedad (mental) es consecuencia directa de los cambios culturales que se van produciendo a lo largo de la historia. Que tanto las prácticas psiquiátricas como los discursos teóricos (o ateóricos) que las sustentan son inseparables de su momento histórico. Esto tiene mucha importancia porque nos permite superar el corsé con que el positivismo científico ha llegado a aprisionar a la locura pero, sobre todo, comprender que los elementos culturales 
ORIGINALES Y REVISIONES

pueden ser cruciales en la aparición de determinados síntomas, lo que obviamente debe tener trascendencia en la clínica.

Pero no solo es preciso tener en cuenta los elementos culturales que pivotan en la génesis de la psicosis o de los trastornos mentales más graves, en los que la "violencia del diagnóstico" y la estigmatización del sujeto siguen formando parte del proceso de inventar/construir a la persona. También en el momento actual, nos explica Fernando Colina, "la sociedad de consumo indujo unas estrategias del deseo exigentes e insaciables, cuya primera consecuencia es la inestabilidad psicológica, la ansiedad y esa intolerancia al duelo, la depresión y la frustración que tan acertadamente nos caracteriza. Una vez instaurado el derecho a la felicidad como una exigencia irreemplazable, cualquier fallo, lentitud o tropiezo del deseo nos vuelve pacientes de la psiquiatría con excesiva facilidad" (93).

No cabe duda que la manera (cultural) de entender el malestar, ha traído consecuencias, sobradamente conocidas, no solo en el ejercicio de la psiquiatría y la psicología (94), sino en una suerte de psiquiatrización de la vida cotidiana (95) sobre las que, no obstante, conviene seguir reflexionando. La obra de Ian Hacking nos puede ofrecer, desde una perspectiva más filosófica que psicopatológica, elementos de discusión importantes que profundicen en la historia cultural del conocimiento psiquiátrico.

\section{BIBLIOGRAFÍA:}

(1) Fleck L. Entstehung und Entwicklung einer wissenschaftlichen Tatsache: Einführung in die Lehre vom Denkstil und Denkkollektiv. Basilea: Benno Schwabe. [trad. cast.: La génesis y el desarrollo de un hecho científico, Madrid: Alianza, 1986].

(2) Arrizabalaga J. La teoría de la ciencia de Ludwik Fleck (1896-1961) y la historia de la enfermedad, Dynamis 1987-88; 7-8: 473-481.

(3) Cunningham A. Transforming plague: the laboratory and the identity of infectious disease. En:Cunningham A, Perry W, editors. The Laboratory Revolution in medicine, Cambridge: Cambridge University Press, 1992; p. 209-244;

(4) Jordanova L. The Social Construction of Medical Knowledge. Social History of Medicine 1995; 8: 361-382.

(5) Arrizabalaga J. Nuevas tendencias en la historia de la enfermedad. Arbor 1992; 142: $147-165$.

(6) Rosenberg Ch E. Disease in History: Frames and Framers. Milibank Quaterly 1989; 67 (suppl. 1): 1-15.

(7) Arrizabalaga J. La peste de 1348: los orígenes de la construcción como enfermedad de una calamidad social. Dynamis 1991; 11: 73-117.

(8) Fee E, Fox D, editors. AIDS. The burdens of history. Berkeley: University of California Press, 1988. 
(9) Figlio K. Chlorosis and chronic disease in nineteenth-century Britain: the social constitution of somatic illness in a capitalist society. Social History 1978; 3: 167-197.

(10) Carrillo JL, Bernal E, Carrillo-Linares JL. Medicina vs mujeres. La literatura médica sobre la clorosis (siglos XVII-XX) ¿ciencia o propaganda?.Málaga: Universidad de Málaga, 2010.

(11) Arrizabalaga J, Herderson J, French R. The Great Pox. The French disease in Renaissance Europe, New Haven: Yale University Press, 1997.

(12) Foucault, M. Folie et déraison. Histoire de la folie à l'âge classique. Paris: Plon, 1961.

(13) Szasz Th. The myth of mental illness; foundations of a theory of personal conduct. New York: Hoeber-Harper, 1961.

(14) Rosen G. Mandess in society. Chapters in the Historical Sociology of Mental Illness. London: Routledge \& Kegan Paul, 1968.

(15) Gallison P. Ten problems in History and Philosophy of Science. Isis 2008; 99: 111-124.

(16) Hacking I. Why Language Matters to Philosophy? Oxford: OUP, 1975.

(17) Hacking I. Representing and Intervining. Introductory Topics in the Philosophy of Natual Science, Cambridge: Cambridge University Press, 1983. [edición en castellano: Representar e intervener. México: Paidos-UNAM, 1996).

(18) Hacking I. Rewriting the Soul: Multiple Personality and the Sciences of Memory. Princenton: Princenton University Press, 1995.

(19) Hacking I. Mad Travelers. Reflections on the Reality of Transient Mental Illnesses. Charlottesville \& London: University Press of Virginia, 1998.

(20) Moscoso, J. Realidad o elaboración de la enfermedad mental. Frenia 2001; 1 (2): 131-144.

(21) Hacking I. The Social Cosntruction of What?. Cambridge: Harvard University Press, 2000 [edición en castellano: ¿La construcción social de qué?, Barcelona: Paidos, 2001]

(22) Martínez ML. Hacking y Latour: realismo y constructivismo. En: Otero, M, editor Constructivismo y realismo. Montevideo: Fundación de Cultura Universitaria, 2000; p. 217-245.

(23) Martínez ML. El realismo científico de Ian Hacking: de los electrones a las enfermedades mentales transitorias. Redes 2005; 11: 153-176.

(24) Hacking I. Historical Onthology. London: Harper University Press, 2002.

(25) Tissié P. Les Aliénés voyageurs. Paris: Doin, 1887.

(26) Beaune JC. Le vagabond et la machine. Essai sur l'automatisme ambulatoire. Médecine, technique et société en France 1880-1910. Seyssel: Champ-Vallon, 1987.

(27) Voisin J. Fugues inconscientes chez les histériques. Semaine médicale $1889 ; 9: 291$.

(28) Pitres A. Leçons cliniques sur l'histérie et l'hypnotisme faites à l'hôpital Saint-André à Bodeaux. Paris : Doin, 1891.

(29) Charcot JM. Accés d'automatisme ambulatoire de nature comitiale", Bulletin de Médicine $1889 ; 3: 275-276$.

(30) Sous MG. De l'automatisme comitiale ambulatoire. Paris: Henri Jouve, 1890.

(31) Dubourdieu F. De la Dromomanie des dégénerés, Thèse. Bordeaux, 1894.

(32) Gilles de la Tourette A. L'automatisme ambulatoire au point de vue médico-légale. Bulletin de Médicine $1889 ; 3: 344$.

(33) Duponchel, E. Étude clinique et médico-légale des impulsions morbides à la déambulation observés chez des militaires. Annales d'hygiène publique et de médecine légale $1888 ; 3: 5-26$;

(34) Denommé P. Des impulsions morbides à la déambulation au point de vue médico-légale. Lyon: Storck, 1894. 
ORIGINALES Y REVISIONES

(35) Cartwright SA. Report on the Diseases and Physical Peculiarities of the Negro Race. The New Orleans Medical and Surgical Journal 1851: 691-715.

(36) Chorover S. Mental health as a social weapon. En: Richardson, H, editor. New Religions and Mental Health: Understanding the Issues. N. York: Edwin Mellen Press, 1980; p. 14-19.

(37) Zárate MS. Dar a luz en Chile, siglo XIX. De la "ciencia de la hembra" a la ciencia obstétrica. Santiago de Chile: Dirección de Bibliotecas, Archivos y Museos, 2007.

(38) Vaschetto E. Aportes al estudio de las locuras puerperales en la Argentina. Temas de Historia de la Psiquiatría Argentina 2009; 28: 27-32.

(39) Voisin A. Leçon cliniques sur les maladies mentales et sur les maladies nerveuses professées a la Salpêtriere. París: J.B. Baillière et fils, 1883.

(40) Huertas R. Cuerpo visto, cuerpo sentido: de la anatomía a la clínica psiquiátrica. Revista de la Asociación española de Neuropsiquiatría 2003; 23 (88): 111-126.

(41) Esquirol JED. Monomanie. En: Dictionnaire des sciences médicales, par une société des médecins et des chirurgiens, Paris, Panckoucke, 1819 ; t. 34 : 114-125.

(42) Martinez-Pérez J. Catalogando la diversidad del comportamiento humano: la nosología francesa decimonónica ante las conductas delictivas (1800-1855). Asclepio 1996; 48 (2): 87-114.

(43) Maristany L. El gabinete del Dr. Lombroso (delincuencia y fin de siglo en España). Barcelona: Anagrama, 1973.

(44) Saussure R de. The influence of the concept of monomania on French medico-legal psychiatry (from 1825-1840). Journal of the History of Medicine and Allied Sciences 1946; 1: 365-397.

(45) Goldstein J. Console ans Classify. The French Psychiatric Profession in the Nineteenth Century. Cambridge: Cambridge University Press, 1987; p. 174-175.

(46) Esquerdo JM. Locos que no lo parecen. Garayo "El Sacamantecas". Madrid: El Liberal, 1981.

(47) Huertas R. Elaborando doctrina: teoría y retórica en la obra de José María Esquerdo (18421912). Frena 2003; 3 (2): 81-109.

(48) Micale MS. Hysteria and Its Historiography: A Review of Past and Present Writings (I y II). History of Science 1989; 27: 223-261; 319-351.

(49) Edelman N. Les métamorphoses de l'hysterique. Du debut du XIXe siècle à la Grande Guerre. Paris : La Découverte, 2003.

(50) Gijswijt-Hofstra M, y Porter R, editors. Cultures of Neurasthenia: From Beard to the First World War. Clio Medica, 63, Amsterdam: Rodopi, 2001.

(51) Martínez-Pérez J. Problemas científicos y socioculturales en la difusión de una doctrina psiquiátrica: la introducción del concepto de monomanía en España”. En: Arquiola, E. y Martínez-Pérez, J editors. Ciencia en expansión. Estudios sobre la difusión de las ideas científicas y médicas en España (siglos XVIII-XX). Madrid: Ed. Complutense, 1995; p. 489-520.

(52) Foucault M. et al. Moi, Pierre Rivière, ayant égorgé ma mère, ma sœur et mon frère...Un cas de parricide au XIXe siècle. Paris : Gallimard, 1973.

(53) Huertas R. Entre la ciencia forense y la legitimación social: En torno al caso Garayo. En: Álvarez, J.M. y Esteban, R, editors. Crimen y locura, Madrid, AEN, 2004; p. 17-34.

(54) Huertas R. Sur les origines de la psychiatrie légale en Espagne. En: Arveiller, J, editor. Psychiatries dans l'histoire. Caen: Press Universitaires de Caen, 2008; p. 375-385.

(55) Campos R. Criminalidad y locura en la Restauración. El proceso del cura Galeote. Frenia 2003; 3 (2): 111-145. 
(56) Campos R, Martínez J, Huertas R. Los ilegales de la naturaleza. Medicina y degeneracionismo en la España de la Restauración (1876-1923). Madrid: CSIC, 2000.

(57) Sérieux P, Capgras J. Les folies raisonantes. Les délires d'interpretation. Paris, Félix Alcan, 1090. [Existe una reciente traducción al castellano -realizada por Ramón Esteban- de esta obra publicada en La Biblioteca de los Alienistas del Pisuerga (Madrid, Ergon, 2007). Presentación, bibliografía y notas de José María Álvarez, Fernando Colina y Ramón Esteban.

(58) Álvarez JM. ¡Qué fue de la paranoia?. En: VV.AA. La Salud Mental en los noventa. Clínica, prácticas, organización. Valladolid: Asociación Castellano-Leonesa de Salud Mental, 1997; p. 43-79.

(59) Álvarez JM. Elogio de la histeria, Cuadernos de Psiquiatría Comunitaria 2006; 6 (2): 111 120.

(60) Álvarez JM, Esteban R, Sauvagnat F. Fundamentos de psicopatología psicoanalítica. Madrid, Síntesis, 2004.

(61) Loewenstein RJ. Psychogenic amnesia and psychogenic fugue: a comprehensive review. Review of Psychiatry 1991; 10: 189-222.

(62) Caro F. Déplacement pathologique: historique et diagnostique différentiels. L'Information Psychiatrique 2006 ; 82 (5) : 405-413.

(63) Moreno Pestaña JL. Los umbrales de entrada en los trastornos alimentarios para las clases populares. Revista Española de Sociología 2005; 5: 25-48.

(64) Brumberg JJ. Fasting Girls. The Emergence of Anorexia Nervosa as a Modern Disease. Londres \& Cambridge: Harvard University Press, 1988;

(65) Walker Bynum C. Holy Feast and Holy Fast: The Significance of Religious Significance of Food to Medieval Women. Berkeley: University of California Press, 1986.

(66) Lasègue Ch. De l'anorexie hysterique. Archives générales de médecine 1873; 21: 385-403. [Una edición en castellano de este artículo puede leerse en la Revista de la Asociación Española de Neuropsiquiatría 2000; 20: 271-282, con una introducción de José María Álvarez (pp. 267-269)].

(67) Gull W. Anorexia nervosa (apepsia hysterica, anorexia hysterica). Transactions of the Clinical Society of London 1874; 7: 22-28.

(68) Ferrús B. Cuerpos que miran a cuerpos. Sobre el imaginario culturista a comienzos del siglo XXI. En: Torras M, editor. Corporizar el pensamiento. Escrituras y lecturas del cuerpo en la cultura occidental, Pontevedra: Mirabel, 2006; p. 115-126.

(69) Campos Rodríguez JM. Anorexia, bulimia e internet. Una aproximación al fenómeno proana y mía desde la teoría subcultural. Frenia 2007; 7 : 127-144.

(70) Voisin J. Automatisme ambulatoire chez une hystérique, avec crises de sommeil. Dédoublement de la personnalité. Annales médico-psychologiques 1889; 10: 418-427.

(71) Hacking I. Macking Up People. En: Heller, T, editor. Reconstructing Individualism: Autonomy, Individuality in Self Investigation Thought, Stanford: Stanford University Press, 1986; p. 161-171.

(72) Hacking I. Looping Effects of Human Kinas. En: Sperber D, Premarck D, Premarck AJ, editors. Causal Cognition: A Multidisciplinary Approach, Oxford, Clarendon Press, 1994.

(73) Tsou JY. Hacking on the Looping Effects of Psychiatric Classifications: What Is an Interactive and Indifferent Kind?. International Studies in the Philosophy of Science 2007; 21 (3): 329-344.

(74) Goffman E. Stigma: Notes on the Management of Spoiled Identity. London: Pelican Books, 1968.

(75) Cura, M del. Los "niños anormales" en la España del primer tercio del siglo XX: la construcción médico-pedagógica de una nueva categoría infantil. En: Perdiguero, E, editor. Salvad al niño. 
ORIGINALES Y REVISIONES

Estudios sobre la protección a la infancia en la Europa Mediterránea a comienzos del siglo XX, Valencia, Seminari d'Estudis sobre la Ciéncia, 2004; p. 273-299.

(76) Hacking I. Philosophie et Histoire des concepts scientifiques. Cours au Collage de France 2004-2005 (dactilografiado).

(77) Bourguignon E. Multiple personality, possession trance, and the psychic unity of mankind. Ethos 1989; 17: 371-384.

(78) Putman FW, Guroff JJ, Silberman EK, Barban L, Post RM. (1986), “The clinical phenomenology of multiple personality disorder: review of 100 recent cases. Journal of Clinical Psychiatry 1986; 47 (6): 285-293.

(79) Kluft RP, Braun BG, Sachs R. Multiple Personality, intrafamilial abuse, and family Psychiatry. American Journal of Family Psychiatry 1984; (5): 283-301.

(80) Kluft RP, editor. Childhood antecedents of Multiple Personality. Washington D.C: American Psychiatric Press Inc, 1990.

(81) Summit RC. The child abuse accomodation síndrome, Child Abuse \& Neglect 1983; 7: $177-$ 193.

(82) Gardner R. Recent trends in Divorce and Custody Litigation. Academy Forum 1985; 29 (2): 3-7.

(83) Escudero A, Aguilar L, Cruz J de la. La lógica del síndrome de alienación parental de Gardner (SAP): terapia de la amenaza. Revista de la Asociación Española de Neuropsiquiatría 2008; 28: 283305.

(84) La Asociación Española de Neuropsiquiatría hace la siguiente declaración en contra del uso clínico y legal del llamado Síndrome de Alienación parental. Revista de la Asociación Española de Neuropsiquiatría 2010; 30: 535-538.

(85) Hacking I. Between Michel Foucalt and Enving Goffman: between discours in the abstract and face-to-face interaction. Economy and Society 2004; 33 (3): 277-302.

(86) Clérambault G G. de. Passion érotique des étoffes chez la femme. Archives d'Anthropologie criminelle, de médecine légale et de psychologie normale et pathologique 1908 ; $25: 439-470$.

(87) Clérambault G G. de. Passion érotique des étoffes chez la femme (suite). Archives d'Anthropologie criminelle, de médecine légale et de psychologie normale et pathologique $1910 ; 27$ : 583-589.

(88) Esteban R, Carreño J. Para una lectura no morbosa de 'Passion érotique des étoffes chez la femme' (1908-1910) de G.G. de Clérambault. Frenia 2006; 7: 127-178.

(89) Lasègue Ch. Vol aux étalages. Archives générales de médecine, 1880. [Reproducido en Lasègue Ch. De la folie à deux à l'hysterie et autres états, París, L'Harmattan, 1998; p. 115-125].

(90) Álvarez JM, Colina F. Las voces y su historia: sobre el nacimiento de la esquizofrenia”, Átopos 2007; 6: 4-12.

(91) Álvarez JM, Colina F. Origen histórico de la esquizofrenia e historia de la subjetividad. En: Martínez Azumendi O, Sagasti N, Villasante O, editores. Del pleistoceno a nuestros días. Contribuciones a la historia de la psiquiatría, Madrid: AEN, 2011; p. 137-148.

(92) Novella EJ, Huertas R. El síndrome de Kraepelin-Bleuler-Schneider y la conciencia moderna: Una aproximación a la historia de la esquizofrenia. Clínica y salud. Investigación empírica en psicopatología 2010; 21 (3): 205-219.

(93) Colina F. Prólogo: Psiquiatría y cultura. En: Álvarez, JM. La invención de las enfermedades mentales. Madrid: Gredos, 2008. 
(94) Ortiz A, García Moratalla B, Mata I de la (2003), "La gestión del malestar en el centro de salud mental", Clínica y pensamiento 2003; 2: 77-86.

(95) Mata I de la, Ortiz A. (2007), "La colonización psiquiátrica de la vida", Archipiélago 2007; (76): $39-50$. 